\title{
Monitoreo de la Savia del Peciolo de Vegetales ${ }^{1}$
}

\author{
George Hochmuth. Traducción por Maria Paz Kinslow²
}

Varios kits de "prueba rápida" de nitrato y potasio para el monitoreo de la savia de vegetales han sido calibrados para su uso en vegetales en la Florida. El objetivo ha sido encontrar un sistema que los productores puedan usar en el campo para ayudar a manejar fertilizantes de nitrógeno $(\mathrm{N})$ y potasio $(\mathrm{K})$, especialmente para vegetales de riego por goteo.

Durante la temporada se prefiere muestrear la planta en lugar del suelo porque, como depósito final de $\mathrm{N}$ y K, la planta provee información para diagnosticar problemas. Además, el N y K son móviles en los suelos arenosos de Florida. Una prueba de suelo para estos nutrientes proporciona solo un vistazo del contenido de nutrientes del suelo, cuyos resultados pueden cambiar rápidamente por la lluvia o el riego.

A medida que los productores y consultores comienzan a usar la tecnología de monitoreo de savia, surgen preguntas sobre los procedimientos del monitoreo de savia. Las siguientes guías deberían ayudar a las personas que actualmente usan o están interesadas en usar el monitoreo de savia. La mayoría de estas guías se han desarrollado a través de investigaciones en Florida, o se basan en la experiencia de campo. Los kits de prueba rápida de savia de plantas miden nitrato-nitrógeno, no $\mathrm{N}$ total.

\section{Cálculos}

Conversiones de nitrato. Algunos kits dan resultados en nitratos y otros en nitrato-nitrógeno. La mayoría de las tablas de calibración están en valores de nitrato-nitrógeno. Para los kits que dan resultados en nitratos $\left(\mathrm{NO}_{3}\right)$, el resultado se debe dividir entre 4.43 para encontrar el valor de nitrato-nitrógeno, que luego se puede comparar con los valores de la tabla. El potasio generalmente se lee directamente como ppm de $\mathrm{K}^{+}$.

Savia vs. peciolos secos. Hay algunos valores en libros publicados para nitrato-nitrógeno del peciolo, pero estos valores a veces se basan en peciolos secos y no se pueden transformar directamente a concentraciones de nitrato-nitrógeno de savia fresca. Sólo valores de nutrientes de la savia de peciolo fresco pueden usarse con un procedimiento de monitoreo de savia del peciolo fresco.

\section{Muestreo}

Hora del día. La temperatura y la hora del día pueden influir en el contenido de nitrato en la savia de la planta. Hacer lecturas consistentemente entre las 9 a.m. y las 4 p.m. dará los resultados más consistentes. La estandarización razonable por temperatura y condiciones climáticas bajo las cuales se realiza el muestreo ayudará a proporcionar resultados más consistentes.

Edad de la hoja. Las tablas de calibración de Florida para el monitoreo de la savia vegetal se desarrollaron para

1. Este documento es CIR1144s, uno de una serie del Horticultural Sciences Department, UF/IFAS Extension. Apoyado en parte por USDA-ES Smith-Lever 3(d) Water Quality Program Support. Publicación original octubre 1994. Revisado abril 2015. Traducido abril 2020. Visite el sitio web de EDIS https:// edis.ifas.ufl.edu.

2. George Hochmuth, profesor emérito, Fertilidad del Suelo y Nutrición Vegetal, Department of Soil and Water Sciences, UF/IFAS Extension, Gainesville, FL 32611. Traducción por Maria Paz Kinslow.

The Institute of Food and Agricultural Sciences (IFAS) is an Equal Opportunity Institution authorized to provide research, educational information and other services

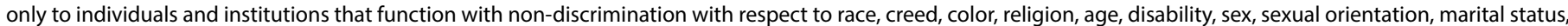

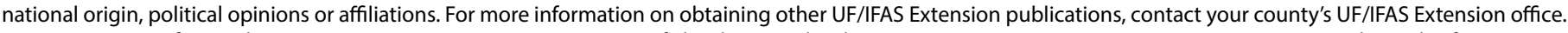
U.S. Department of Agriculture, UF/IFAS Extension Service, University of Florida, IFAS, Florida A \& M University Cooperative Extension Program, and Boards of County Commissioners Cooperating. Nick T. Place, dean for UF/IFAS Extension. 
los peciolos (tallos de las hojas) de las hojas que hayan madurado recientemente (MRML, por sus siglas en ingles), aquellas hojas que han alcanzado el tamaño máximo (esencialmente dejaron de expandirse). Las hojas MRML también han cambiado de un color verde claro juvenil a un color verde oscuro.

Parte de la hoja. Las pruebas se calibraron utilizando el peciolo grueso de la hoja (Figura 1). En la mayoría de los cultivos, el peciolo es fácil de identificar. Para los tomates, que tienen hojas compuestas, el peciolo es el tallo entero con todos los pequeños peciolos (y pequeños folíolos) removidos. En situaciones normales el peciolo de la hoja de tomate tendrá aproximadamente 8 pulgadas de largo. Los peciolos de la hoja de chile tienen sólo una pulgada de largo.

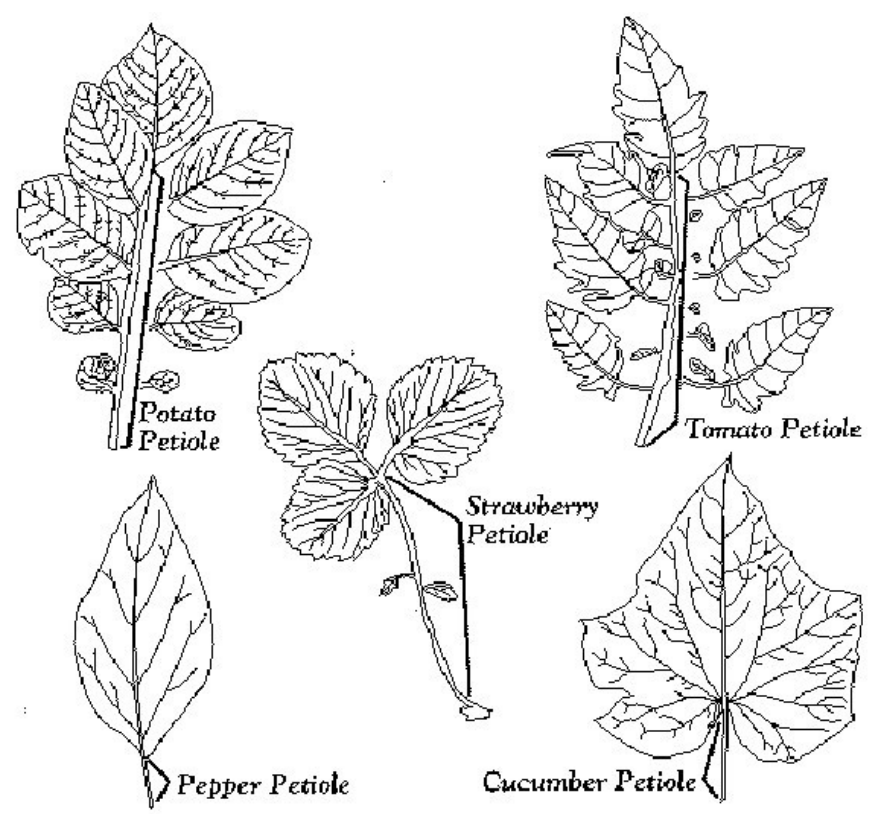

Figura 1. Potato petiole=peciolo de papa; tomato petiole=peciolo de tomate; strawberry petiole=peciolo de fresa; pepper petiole=peciolo de chile; cucumber petiole=peciolo de pepino.

Número de hojas. Aunque tres o cuatro peciolos pueden producir una cantidad suficiente de savia para el monitoreo, se deben tomar muestras de plantas adicionales para garantizar que la muestra de savia sea representativa del campo o área que se está monitoreando. Por lo general, alrededor de 20 hojas son suficientes para representar adecuadamente un campo de 5 a 10 acres, si se considera que ese campo es uniforme. Los peciolos se deben cortar y mezclar, y una submuestra de las piezas de peciolo picado utilizadas para la muestra final para prensar.

\section{Monitoreo de Savia}

Equipo. Se utiliza una prensa de ajo o limón para exprimir la savia de los pedazos de peciolo. Si se harán muchas muestras, es útil una prensa hidráulica de savia. Una prensa también es útil para los peciolos que tienen poca savia, por ejemplo, fresa o chile. Otro equipo incluye un cuchillo de muestreo, tijeras, toallas de papel, agua destilada, cuchillo y tabla para picar, y los kits de prueba. Hay kits de prueba separados para $\mathrm{N}$ y K.

Almacenamiento de peciolos. Se han realizado estudios en Florida para probar opciones para almacenamiento de muestras de peciolos para determinar la flexibilidad de las metodologías de monitoreo para aquellos que desean monitorear varias granjas antes de realizar las pruebas de savia. Los peciolos frescos, enteros (sin cortar) pueden almacenarse en hielo hasta 8 horas o congelarse durante la noche sin cambios apreciables en las concentraciones de $\mathrm{N}$ o K en la savia. Se deben remover las hojas de los peciolos y colocarlos en una bolsa de plástico sobre hielo en un refrigerador.

Los peciolos pueden almacenarse a temperatura ambiente $\left(70^{\circ} \mathrm{F}\right)$ en una bolsa de plástico hasta 2 horas. Si las hojas enteras o los peciolos se almacenan al aire libre, los peciolos se marchitarán y las lecturas de nutrientes no serán precisas. Sólo deben almacenarse los peciolos, no la savia. Los peciolos fríos deben regresarse a temperatura ambiente antes de prensarlos, para que las diferencias de temperatura entre la savia y el medidor no afecten los resultados.

Marco de tiempo de lectura. La medida del contenido de nutrientes de la savia prensada debe realizarse dentro de uno o dos minutos después del prensado. De lo contrario, las lecturas de nitrato podrían cambiar de la condición de peciolo fresco cuando la savia se expone al aire.

\section{Manejo del Kit de Prueba}

Los kits de prueba deben calibrarse y probarse con soluciones estándar, conocidas de nitrato y $\mathrm{K}$, disponibles del fabricante del kit de prueba. Con los kits de prueba colorimétricas, la calibración con soluciones conocidas revelará si los productos químicos todavía están buenos. Los productos químicos en tiras reactivas o en almohadillas en polvo se deteriorarán con el tiempo y a al ser expuestos al calor y la luz.

Los kits de prueba de electrodos deberán calibrarse con frecuencia con soluciones estándar. La calibración debe verificarse cada cinco o seis muestras. Las lecturas deben 
hacerse en la sombra o en un laboratorio, ya que la luz solar directa en el medidor puede afectar su funcionamiento.

Para la prueba de tiras Quant (Figura 2), se retira una tira de prueba del recipiente (mantenga las tiras frías cuando no estén en uso) y se sumerge por un segundo en la savia diluida. Después de 60 segundos, el color rosa o morado desarrollado en la porción de prueba en el extremo de la tira se compara con la tabla de colores calibrada provista con el kit. La interpolación es necesaria para lecturas entre dos bloques de color en la tabla. Una alternativa es usar un lector de color de banda recientemente desarrollado. Este reflectómetro proporciona una evaluación más cuantitativa del color en la tira. Las lecturas se realizan en partes por millón (ppm) de nitratos que se pueden convertir en ppm de nitrato- $\mathrm{N}$ dividiendo entre 4.43 .

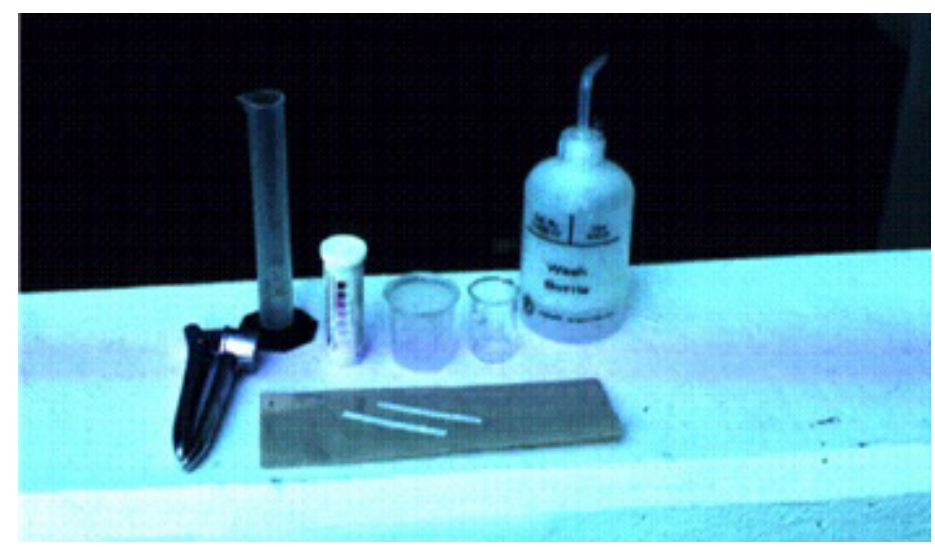

Figura 2.

Para el colorímetro $\mathrm{HACH}$, dos tubos de observación se llenan con savia diluida (Figura 3). Se coloca un tubo en su ranura en el "comparador". El contenido de una almohadilla de reactivo en polvo se vacía en la segunda muestra de savia diluida y el tubo se mezcla durante un minuto. Después de mezclar, el tubo se coloca en su ranura en el "comparador" y se deja durante un minuto. Después de un minuto, los colores en las ranuras de observación se emparejan girando la rueda de colores, y las ppm resultantes de nitrato- $\mathrm{N}$ se leen del dial.

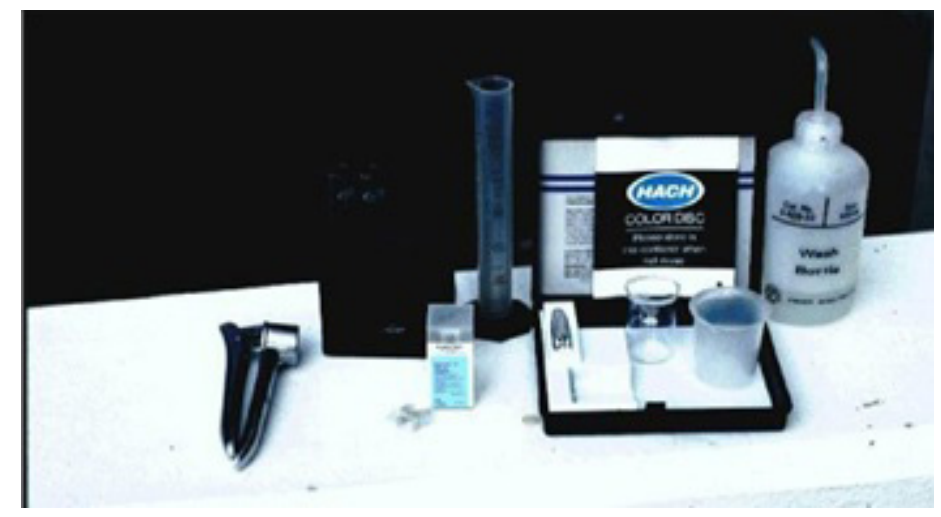

Figura 3.
Los métodos colorimétricos pueden estar influenciados por la coloración de clorofilas en la savia. La filtración con carbón de la savia diluida antes de la lectura podría mejorar la precisión de la medición de nitrato-N en savia con mucho color.

Para los medidores Cardy (medidor separado para análisis de $\mathrm{N}$ y K), la savia extraída se coloca en los electrodos calibrados para que una película cubra ambos electrodos continuamente (Figura 4). La concentración de nitratos o K se lee en la balanza digital que cambia automáticamente entre escalas 1x, 10x o 100x, dependiendo de la concentración de nutrientes en la savia. Los medidores deben usarse y almacenarse en un ambiente fresco y seco. Los electrodos se pueden reemplazar por alrededor de $\$ 50$ y generalmente son buenos para hasta 500 medidas.

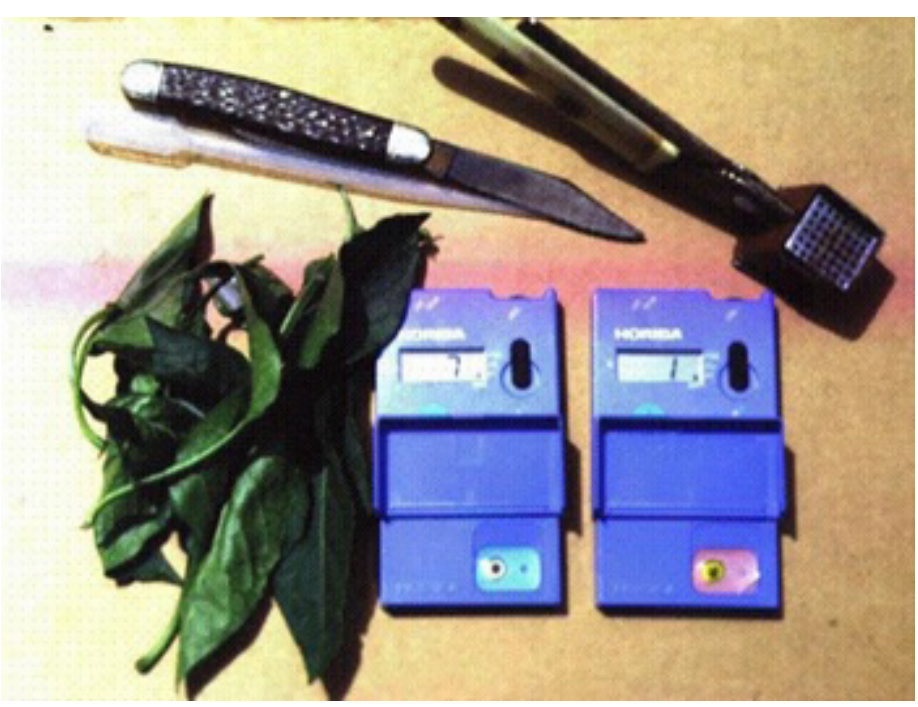

Figura 4.

Escala de calibración. Las muestras siempre deben leerse dentro de la escala de calibración (lectura) del instrumento del kit de prueba. Las lecturas fuera del rango calibrado deben considerarse inexactas. Si las concentraciones de nutrientes de la savia de la muestra son más altas que el extremo superior de la escala de calibración, la savia debe diluirse. La dilución se puede hacer con agua sin nitrato usando aproximadamente 20 a 50 partes de agua por 1 parte de savia.

Cuidado del kit. Los kits de prueba de savia son herramientas científicas que requieren un tratamiento cuidadoso. Los kits y productos químicos deben almacenarse en un lugar protegido, dentro de los rangos de temperatura adecuados especificados por el fabricante. Los kits no deben almacenarse en una camioneta o estación de bombeo entre usos. 


\section{Recomendaciones}

Guías para las concentraciones de $\mathrm{N}$ y K de savia del peciolo se presentan en la Tabla 1. Los rangos presentados son valores críticos sugeridos y es posible que sea necesario refinarlos en base a investigaciones futuras o experiencia de campo.

\section{Lectura Adicional}

Coltman, R. R. 1987. "Sampling considerations for nitrate quick tests of greenhouse-grown tomatoes." J. Amer. Soc. Hort. Sci. 112: 922-927.

Fletcher, J., R. Hochmuth, and G. Hochmuth. 1993.

"Calibration of $\mathrm{N}$ and $\mathrm{K}$ fresh sap quick-test procedures for polyethylene-mulched peppers." Proc. 24th Nat'l. Agricultural Plastics Congr. 24: 147-152.

Hochmuth, G., D. Maynard, C. Vavrina, and E. Hanlon. 1991. "Plant tissue analysis and interpretation for vegetable crops in Florida." Fla. Coop. Ext. Serv. Special Series SS-VEC-42.

Hochmuth, G. J., P. R. Gilreath, E. A. Hanlon, G. A. Clark, D. N. Maynard, C. D. Stanley, and D. Z Haman. 1988. "Evaluating plant N status with plant sap quick-test kits." Proceedings of Tomato Institute. Fla. Coop. Ext. Serv. Spec. Series SS-VEC-801:6-14.

Hochmuth, G. J., B. C. Hochmuth, E. A. Hanlon, and M. E. Donley. 1992. "Effect of potassium on yield and leaf-N and K concentrations of eggplant." Suwannee Valley AREC Res. Report 92-2, 16 pp.

Hochmuth, R. C., and G. J. Hochmuth. 1991. "Nitrogen requirements for mulched slicing cucumbers." Suwannee Valley AREC Res. Report 91-18, 8 pp.

Hochmuth, G., B. Hochmuth, E. Hanlon, and M. Donley. 1991. "Nitrogen requirements of mulched eggplant in northern Florida." Suwannee Valley AREC Res. Report 91-14, 13 pp.

Prasad, M., and T. M. Spiers. 1984. "Evaluation of a rapid method for plant sap nitrate analysis." Commun. Soil Sci. Plant Anal. 15:673-679.

Vann, C. D., R. C. Hochmuth, and G. J. Hochmuth. 1993. "Watermelon N and K petiole sap testing." Proc. 1993 Watermelon Institute. Fla. Coop. Ext. Serv. Special Series SS-HOS-003. 
Tabla 1. Guía para el muestreo de nitrato-nitrógeno y potasio de savia fresca del peciolo de la planta.

\begin{tabular}{|c|c|c|c|}
\hline \multirow[t]{2}{*}{ Cultivo } & \multirow[t]{2}{*}{ Etapa de desarrollo de cultivos } & \multicolumn{2}{|c|}{$\begin{array}{c}\text { Concentración (ppm) en savia del peciolo } \\
\text { fresco }\end{array}$} \\
\hline & & $\mathrm{NO}_{3}-\mathrm{N}$ & $\mathbf{K}$ \\
\hline \multirow[t]{3}{*}{ Brócoli y col } & Etapa de seis hojas & $800-1000$ & $N R^{z}$ \\
\hline & Una semana antes de la primera cosecha & $500-800$ & \\
\hline & Primera cosecha & $300-500$ & \\
\hline \multirow[t]{3}{*}{ Pepino } & Primera flor & $800-1000$ & NR \\
\hline & Frutas de tres pulgadas de largo & $600-800$ & \\
\hline & Primera cosecha & $400-600$ & \\
\hline \multirow[t]{3}{*}{ Berenjena } & Primera fruta (dos pulgadas de largo) & $1200-1600$ & $4500-5000$ \\
\hline & Primera cosecha & $1000-1200$ & $4000-5000$ \\
\hline & Mediados de cosecha & $800-1000$ & $3500-4000$ \\
\hline \multirow[t]{3}{*}{ Melón } & Primera flor & $1100-1200$ & NR \\
\hline & Fruta de dos pulgadas de largo & $800-1000$ & \\
\hline & Primera cosecha & $700-800$ & \\
\hline \multirow[t]{5}{*}{ Chile/Pimiento } & Primeros brotes florales & $1400-1600$ & $3200-3500$ \\
\hline & Primeras flores abiertas & $1400-1600$ & $3000-3200$ \\
\hline & Frutas a medio crecimiento & $1200-1400$ & $3000-3200$ \\
\hline & Primera cosecha & $800-1000$ & $2400-3000$ \\
\hline & Segunda cosecha & $500-800$ & $2000-2400$ \\
\hline \multirow[t]{5}{*}{ Papa } & Plantas de ocho pulgadas de alto & $1200-1400$ & $4500-5000$ \\
\hline & Primeras flores abiertas & $1000-1400$ & $4500-5000$ \\
\hline & $50 \%$ de flores abiertas & $1000-1200$ & $4000-4500$ \\
\hline & $100 \%$ flores abiertas & $900-1200$ & $3500-4000$ \\
\hline & Parte superior cayéndose & $600-900$ & $2500-3000$ \\
\hline \multirow[t]{2}{*}{ Calabaza/Zapallo } & Primera flor & $900-1000$ & NR \\
\hline & Primera cosecha & $800-900$ & \\
\hline \multirow[t]{6}{*}{ Fresa } & Noviembre & $800-900$ & $3000-3500$ \\
\hline & Diciembre & $600-800$ & $3000-3500$ \\
\hline & Enero & $600-800$ & $2500-3000$ \\
\hline & Febrero & $300-500$ & $2000-2500$ \\
\hline & Marzo & $200-500$ & $1800-2500$ \\
\hline & Abril & $200-500$ & $1500-2000$ \\
\hline \multirow[t]{6}{*}{ Tomate (Campo) } & Primeros brotes & $1000-1200$ & $3500-4000$ \\
\hline & Primeras flores abiertas & $600-800$ & $3500-4000$ \\
\hline & Frutos de una pulgada de diámetro & $400-600$ & $3000-3500$ \\
\hline & Frutos de dos pulgadas de diámetro & $400-600$ & $3000-3500$ \\
\hline & Primera cosecha & $300-400$ & $2500-3000$ \\
\hline & Segunda cosecha & $200-400$ & $2000-2500$ \\
\hline \multirow[t]{3}{*}{ Tomate (Invernadero) } & Trasplante al segundo racimo de frutas & $1000-1200$ & $4500-5000$ \\
\hline & Segundo racimo al quinto racimo de frutas & $800-1000$ & $4000-5000$ \\
\hline & Temporada de cosecha (Diciembre a Junio) & $700-900$ & $3500-4000$ \\
\hline \multirow[t]{4}{*}{ Sandía } & Enredaderas de 6 pulgadas de largo & $1200-1500$ & $4000-5000$ \\
\hline & Frutas de 2 pulgadas de largo & $1000-1200$ & $4000-5000$ \\
\hline & Frutas mitad de madurez & $800-1000$ & $3500-4000$ \\
\hline & En la primera cosecha & $600-800$ & $3000-3500$ \\
\hline
\end{tabular}

